\title{
The Pattern Recognition System Using the Fractal Dimension of Chaos Theory
}

\section{Young-Woo Shon}

Department of Multimedia, Kimpo College, Gimpo, Korea

\section{]jfis}

Received: May 31, 2015

Revised : Jun. 24, 2015

Accepted: Jun. 25, 2015

Correspondence to: Young-Woo Shon (ywson@kimpo.ac.kr)

(CThe Korean Institute of Intelligent Systems

cCThis is an Open Access article distributed under the terms of the Creative Commons Attribution Non-Commercial License (http://creativecommons.org/licenses/ by-nc/3.0/) which permits unrestricted noncommercial use, distribution, and reproduction in any medium, provided the original work is properly cited.

\begin{abstract}
In this paper, we propose a method that extracts features from character patterns using the fractal dimension of chaos theory. The input character pattern image is converted into timeseries data. Then, using the modified Henon system suggested in this paper, it determines the last features of the character pattern image after calculating the box-counting dimension, natural measure, information bit, and information (fractal) dimension. Finally, character pattern recognition is performed by statistically finding each information bit that shows the minimum difference compared with a normalized character pattern database.
\end{abstract}

Keywords: Fractal dimension, Modified Henon attractor, Chaos theory, Pattern recognition system, Image processing system

\section{Introduction}

Chaos theory is the study of the irregular, unpredictable behavior of deterministic and nonlinear dynamical systems. Using a chaos interpretation allows us to evaluate the characteristics existing in a system's status space, using a time-series, to extract the chaos characteristic understanding, these characteristics enable us to conduct a high-precision interpretation [1-4].

Therefore, this paper proposes a new method that extracts character features and recognizes characters using the fractal dimension of chaos theory; it precisely recognizes minute differences with a strange attractor created from the Henon system. This paper presents a high-precision character recognition system.

The same kind of difficulties are found in recognizing Hangul (Korean) characters, as are found in English word recognition because Hangul characters have a similar hierarchy graphemes are combined to form characters [5-7]. In order to solve the variability and ambiguity problems, statistical and structural methods have been mainly used in previous studies.

\section{Proposed Character Recognition System}

\subsection{Proposed System}

The proposed system first obtains the mesh, projection and cross-distance features from the character images. The features are converted into time-series data. Then, using the modified Henon system suggested in this paper, each character's attractor for a standard Korean character set, KSC 5601, is reconstructed (Figure 1). 


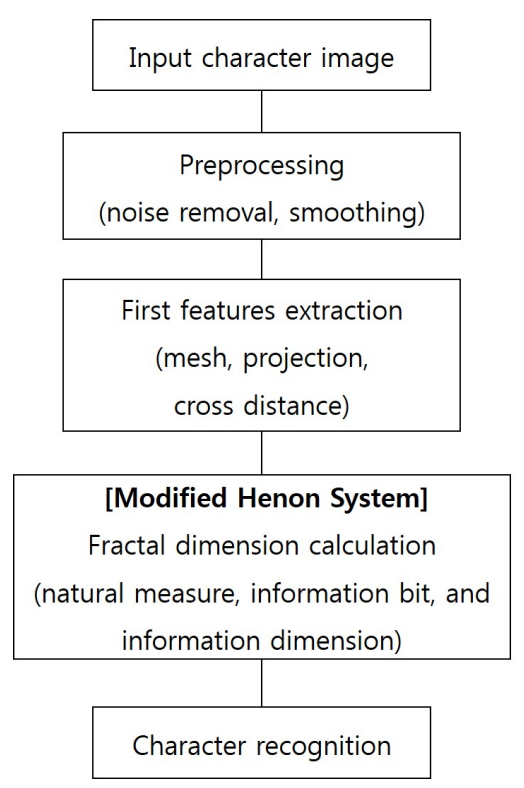

Figure 1. Proposed character recognition system.

\subsection{Henon's Attractor}

Henon's Attractor is a discrete dynamical system in two dimensions. It was suggested by the French astronomer Michel Henon in 1976 as a simplified model for the dynamics of the Lorenz system [8]. Because of its simplicity, it lends itself to computer studies and numerous other investigations. Moreover, the gently swirling, boomerang-like shape of the attractor that arises through the dynamics is aesthetically very appealing. This object is now known as the Henon attractor (Figure 2).

In a manner which we will specify, the Henon system leads from the one-dimensional dynamics of the quadratic transformation to multi-dimensional strange attractors.

The stretch-and-fold action of the Henon system occurs in two dimensions, with coordinates denoted by $x$ and $y$. The transformation, thus, is a plane transformation which operates just like one of the affine transformations from our paradigm [1]. Explicitly, Henon suggested that a transformation is

$$
H(x, y)=\left(y+1-a x^{2}, b x\right)
$$

where $a$ and $b$ are adjustable parameters. An orbit of the system consists of a starting point $\left(x_{0}, y_{0}\right)$ and its iterated image; i.e.,

$$
\begin{gathered}
H\left(x_{k+1}, y_{k+1}\right)=\left(y_{k}+1-a x_{k}^{2}, b x_{k}\right), \\
k=0,1,2,3, \ldots
\end{gathered}
$$

Similar to the logistic equation, these dynamics depend dramat-
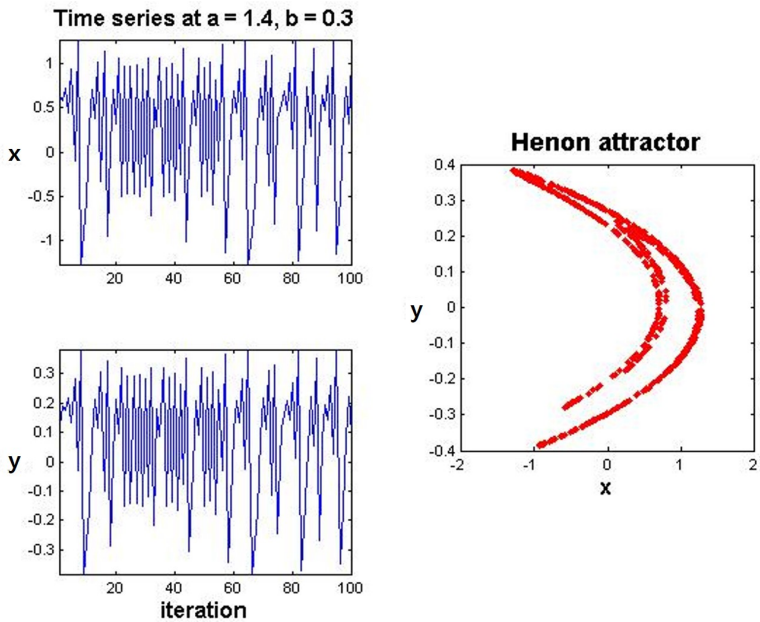

Figure 2. Henon attractor.

ically on the choice of the constants $a$ and $b$, in addition to that of the starting point. For some parameters, almost all orbits tend to have a unique periodic cycle, while chaos seems to reign for other choices, Henon used the values $a=1.4$ and $b=0.3$.

\subsection{Creation of a Character Attractor}

The system extracts the first feature from the input character images, and the feature is converted into time-series data. Then, using the modified Henon system suggested in this paper, the character attractors are reconstructed; i.e.,

$$
\begin{aligned}
& H^{\prime}\left[x_{k}, y_{k}\right] \\
& \quad=\left[y_{k}+1--a\left(x_{k}+\mathbf{c f}_{i}\right)^{2}, b\left(x_{k}+\mathbf{c f}_{i}\right)\right], \\
& \quad k=0,1,2,3, \ldots, n
\end{aligned}
$$

where $\mathbf{c} \mathbf{f}_{i}$ is the first feature of the input character image. In addition, in the experiment, the following Modified Henon system parameters are adopted

$$
a=0.55 \text { and } b=0.3
$$

The best character attracters are reconstructed in this case (Figure 3).

\section{Analysis of a Fractal Dimension}

The concept of a fractal dimension has inspired scientists to produce a host of interesting new work and fascinating speculations. Indeed, for a while, it seemed as if the fractal dimension 


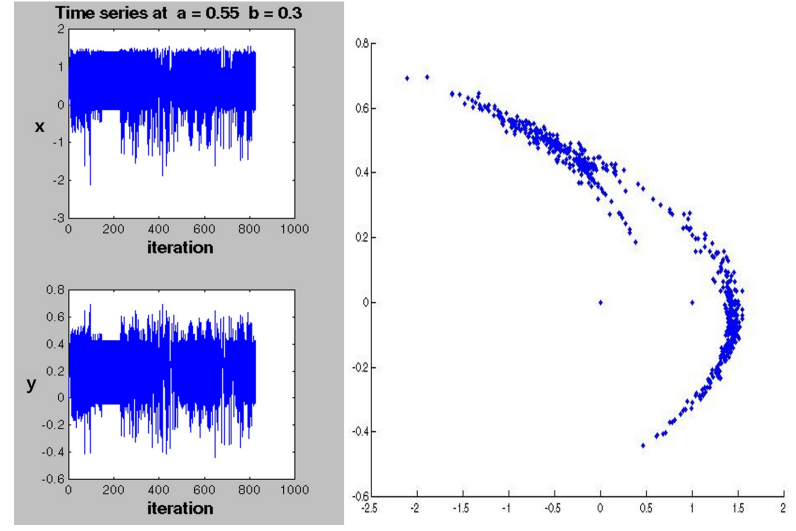

Figure 3. Time series and attractors of a character image using the proposed system.

would allow us to discover a new order in the world of complex phenomena and structures.

In this paper, in order to analyze the chaotic degree of each character's attractor, the system obtains the last features of the character image after calculating the box-counting dimension, the natural measure, the information bit, information (fractal) dimension [8-10].

\subsection{Box-Counting Dimension}

The box-counting dimension is related to the self-similarity dimension and is the most used measurement in all the sciences. The reason for its dominance lies in its easy and automatic computability by machine. It is straightforward to count boxes and to maintain statistics allowing dimensional calculation.

We place the structure on a regular mesh, with mesh, size $s$ (scaling factor), and simply count the number of grid boxes which contain some of the structure. This produces a number, say $N$. Since this number depends on our choice of $s$. we write $N(s)$. Next, we make a log/log-diagram. We then try to fit a straight line to the plotted points of the diagram and measure its slope, $D_{f}$. This number is the box-counting dimension (Eq. (4)).

$$
D_{f}=\lim _{s \rightarrow 00} \frac{\log N(s)}{\log 1 / s}
$$

where, scaling factor $s=1 / 8$.

\subsection{Natural Measure}

To compute the box-counting dimension, we first find a rectangular region that contains the complete attractor. To overcome this shortcoming of the fractal dimension, boxes should be

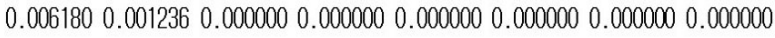

0.0000000 .0271940 .0803460 .1458590 .0123610 .0000000 .0000000 .000000

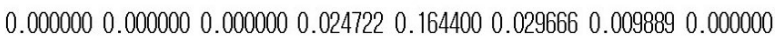

0.0000000 .0000000 .0000000 .0000000 .0012360 .0111250 .0543880 .040791

0.0000000 .0000000 .0000000 .0000000 .0000000 .0024720 .0580960 .252163

0.0000000 .0000000 .0000000 .0000000 .0000000 .0148330 .0494440 .000000

0.0000000 .0000000 .0000000 .0024720 .0074170 .0012360 .0000000 .000000

0.0000000 .0000000 .0000000 .0000000 .0000000 .0000000 .0000000 .000000

Figure 4. Natural measure.

weighted according to how many times an orbit visits them. Consider an open subset $B$ of a space $X$ in which an attractor lies; for example, a subset $B$ of a plane or the Euclidean three-dimensional space. Orbits that are typically observed in computer studies seem to densely fill up the attractor, eventually.

We can count the number of times an orbit $x_{0}, x_{1}, x_{2}, \ldots \in X$ enters subset $B$, and it is natural to assume that the percentage of all points in $B$ stabilizes as we perform more and more iterations. This percentage is called the natural measure, $\mu(B)$, for the system.

Formally,

$$
\left.\mu(B)=\lim _{n \rightarrow \infty} \frac{1}{x+1} \sum_{k=0}^{n} 1_{B}\left(x_{k}, y\right) k\right)
$$

where $1_{B}(x, y)$ is a function which returns 1 or 0 to indicate whether $x$ is in $B$ or not. In other words,

$$
1_{B}(x k, y k)=\left\{\begin{array}{l}
1 \text { if }\left(x_{k}, y_{k}\right) \in B \\
\text { 0otherwise }
\end{array}\right.
$$

and $\sum_{k=0}^{n} 1_{B}\left(x_{k}, y_{k}\right)$ is the number of points from the orbit $x_{0}$, $x_{1}, x_{2}, \ldots, x_{n}$ which fall in the set $B$.

The natural measure can be understood as a means of quantifying the mass of a portion of the attractor. The calculated result of the natural measure is shown in Figure 4.

\subsection{Information Bit and Dimension}

The theoretical foundation was given by Claude Shannon in 1948. The logical approach would be to replace the simple boxcount by a counting procedure in which each box is weighted according to its natural measure. Thus, places which the orbit 
0.0453530 .0119410 .0000000 .0000000 .0000000 .0000000 .0000000 .000000

0.0000000 .1414240 .2922690 .4051020 .0783440 .0000000 .0000000 .000000

0.0000000 .0000000 .0000000 .1319670 .4282160 .1505570 .0658590 .000000

0.0000000 .0000000 .0000000 .0000000 .0119410 .0722010 .2284610 .188275

0.0000000 .0000000 .0000000 .0000000 .0000000 .0214090 .2385090 .501192

0.0000000 .0000000 .0000000 .0000000 .0000000 .0901120 .2144900 .000000

0.0000000 .0000000 .0000000 .0214090 .0524720 .0119410 .0000000 .000000

0.0000000 .0000000 .0000000 .0000000 .0000000 .0000000 .0000000 .000000

Figure 5. Information bit.

passes through very frequently have a stronger impact on the calculation than boxes which the orbit rarely visits. In terms of a formula, we replace $\log N(s)$ by

$$
I(s)=\sum_{k=1}^{N(s)} \mu\left(B_{k}\right) \log _{2} \frac{1}{\mu\left(B_{k}\right)}
$$

Here, the sum ranges over all $N(s)$ boxes $B_{k}$ of linear size $s$ that cover the attractor. The quantity $I(s)$ specifies the amount of information necessary to specify a point of the attractor to within an accuracy of $s$; or, in other words, it is the information obtained in making a measurement that is uncertain by amount $s$. The calculated result of the information bit, $I(s)$, is shown in Figure 5. The information dimension $D_{I}$ is obtained in Eq. (8).

$$
D_{I}=\lim _{s \rightarrow>0} \frac{I(s)}{\log _{2} 1 / s}
$$

\section{Implementation and Experimental}

\subsection{Implementation}

First, using modified the Henon system suggested in this paper, each character's attractor for the standard Korean character set, KSC 5601, is reconstructed. Next, in order to analyze the chaotic degree of each character's attractor, the system finds the last features of the character image after calculating the box-counting dimension, natural measure, information bit, information (fractal) dimension.

Even though calculating of the box-counting dimension for a character attractor in character recognition is very simple, it only determines whether the attractor exists in a fixed box size.

Thus, is very sensitive to noise and does not well reflect the characteristic information of the character. Therefore, after using the information dimension and information bit computed based on the natural measure, which shows the characteristic in-

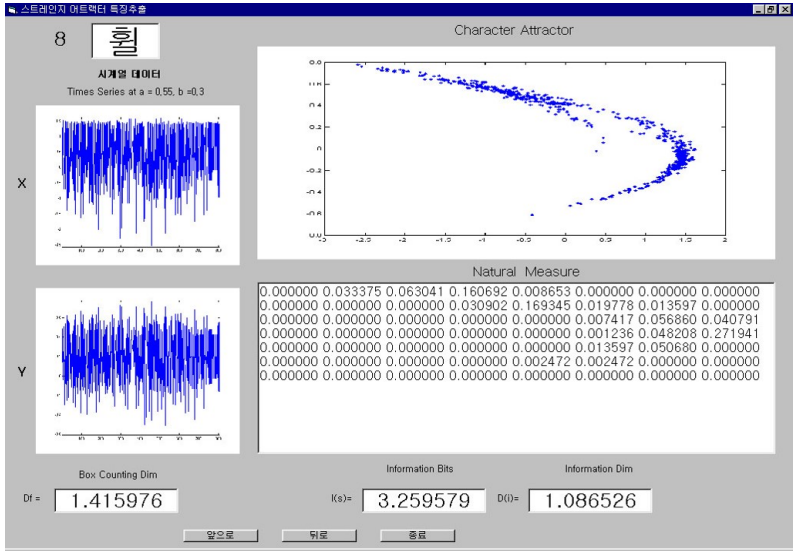

Figure 6. Fractal dimension at $s=1 / 8$.

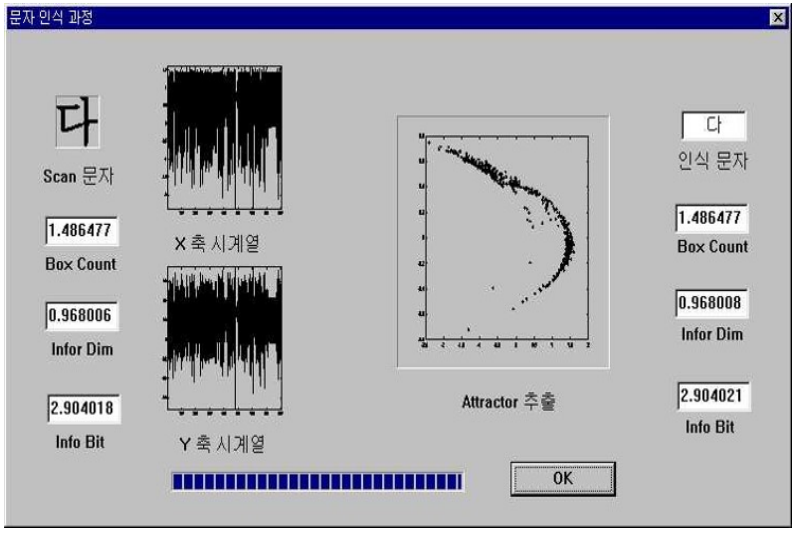

Figure 7. Character recognition process for the proposed system.

formation of character, we do global and detailed classifications by means of a statistical method using the minimum distance value of the characteristic vector.

\subsection{Results and Analysis}

In the experiment, we adopted Henon system parameters ( $a=0.55, b=0.3)$ to reconstruct the best character attractor and adopt the proper box-count size for seeking the best fractal dimension characteristic value, in behalf of high-precision character recognition from a character attractor. Further, we developed a strange attractor simulator in order to quantitatively discriminate the chaos status. With time-series data as analysis input, this simulator automatically reconstructed the attractor in state space and automatically calculated its dimension at high speed.

The calculated result of the fractal dimension is shown in Figure 6 and the character recognition process for the proposed 
system is shown in Figure 7.

\section{Conclusions}

This paper proposed a new method which extracts character features and recognizes characters using the fractal dimension of chaos theory which accurately recognizes minute differences with a strange attractor created with the Modified Henon system. This paper implements a high-precision character recognition system.

\section{Conflict of Interest}

No potential conflict of interest relevant to this article was reported.

\section{Acknowledgements}

This work was supported by the 2015 Research Fund of Kimpo College, Korea.

\section{References}

[1] H. O. Peitgen, H. Jurgens, and D. Saupe, Chaos and Fractals: New Frontiers of Science. New York, NY: Springer, 1992.

[2] E. Ott, Chaos in Dynamical Systems. Cambridge; Cambridge University Press, 1993.

[3] S. H. Kellert, In the Wake of Chaos: Unpredictable Order in Dynamical Systems. Chicago, IL: University of Chicago press, 1993.

[4] J. Gleick, Chaos: Making a New Science. London: Abacus, 1993.
[5] M. Nadler and E. P. Smith, Pattern Recognition Engineering. New York, NY: Wiley, 1993.

[6] K. S. Fu, Syntactic Methods in Pattern Recognition. New York, NY: Academic Press, 1974.

[7] Y. W. Shon and J. C. Namkung, "A study on the Hangeul confusion character recognition using fractal dimension and attractor," The Transactions of the Korea Information Processing Society, vol. 6, no 7, pp. 1825-1831, 1999.

[8] M. Henon, "A two-dimensional mapping with a strange attractor," Communications in Mathematical Physics, vol. 50, no. 1, pp. 69-77, 1976. http://dx.doi.org/10.1007/ BF01608556

[9] W. Ko, "Dynamic system, chaos, fractal," May 2012. http: //blog.daum.net/w-ko/129.

[10] G. Jin, "Triangular prism method based on an enhanced sampling method," Journal of Korean Institute of Intelligent Systems, vol. 23, no. 2, pp. 93-99, 2013. http://dx.doi.org/ 10.5391/jkiis.2013.23.2.93

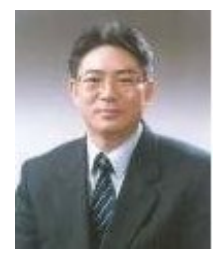

Young-Woo Shon received his B.S., M.S., and Ph.D. from the Department of Computer Engineering, Kwangwoon University, Korea, in 1981, 1983 and 2000, respectively. $\mathrm{He}$ had worked at Korea Institute of Science and Technology Information (KISTI) during 1991-1998 as a senior researcher. He is currently a professor at the Department of Multimedia, Kimpo College, Kimpo, Korea. His research interests include Chaos Theory, Pattern Recognition, Artificial Intelligent System. He is a member of KISS, KIECS and KMMS

E-mail: ywson@kimpo.ac.kr 